\title{
CONVIVENCIA ESCOLAR: UNA MIRADA DESDE LAS FAMILIAS
}

\author{
School coexistence: A look from the families
}

\author{
Luz S. Fuentes $\mathrm{F}^{*}$ y Libia M. Pérez $\mathrm{C}^{* *}$
}

\section{RESUMEN}

El presente trabajo tuvo como objetivo describir la percepción sobre convivencia escolar desde la visión de las familias de los estudiantes. Para ello se retomó como soporte teórico autores como Colombo (2011), Chaux (2013), Zaitegui (2006), Ramírez (2016), López de Mesa, Et al., (2013) y Valdés y Urías (2010). En cuanto a los aspectos metodológicos se adaptaron los instrumentos para la recolección de datos propuestos por Ortega y Del Rey (2002). La muestra es no probabilística, los criterios de inclusión fueron ser padre de familia de estudiantes matriculados de primero a quinto grado de una institución oficial municipal en Sincelejo-Colombia, y asistir a una convocatoria general realizada, donde hizo acto de presencia sólo 58 representantes de familias quienes se constituyen en la muestra. El análisis de datos se realizó mediante el uso de estadística descriptiva. Los resultados muestran una percepción favorable en la relación entre profesores y estudiantes. Mientras tanto, entre estudiantes la situación es diferente, pues se presenta un alto porcentaje de peleas, apodos e insultos. Además, la exclusión y el irrespeto a las normas, son

\footnotetext{
* Licenciada en Pedagogía Reeducativa de la Fundación Universitaria Luis amigó Montería, Magister en Educación y Cognición de la Universidad del Norte Barranquilla, Doctora en Educación de la Universidad Privada Dr. Rafael Belloso Chacín. Investigador por categorizar en Colciencias. Docente- investigador del grupo: REDINA. Corporación Universitaria del Caribe -CECAR, Sincelejo - Colombia. Correo: luz.fuentes@ cecar.edu.co_ ORCID ID. 0000-0001$8257-4814$

** Bióloga con énfasis en Biotecnología de la Universidad de Sucre, Magister en ciencias ambientales del Sistema de las Universidades Estatales de la Costa Caribe (SUECcaribe), Doctora en Educación de la Universidad Privada Dr. Rafael Belloso Chacín. Investigador del grupo DIDACINNOVACIONCN. Universidad Popular Del Cesar - Upc- Colombia. Correo: libiamariaperez@hotmail.com
} 
situaciones que desfavorecen el clima escolar, sin embargo, la mayoría de los conflictos están asociados a un grupo reducido de estudiantes con conductas disruptivas violentas y malos tratos, lo cual afecta el clima de aula. Ante estas situaciones, las familias sugieren la necesidad de implementar una estrategia de mejoramiento integral de la convivencia que fortalezca las competencias ciudadanas para así, garantizar una calidad de la convivencia escolar institucional.

Palabras Clave: Convivencia, Escuela, Familia, Conflicto.

RECIBIDO: Agosto 2018

ACEPTADO: Noviembre 2018

\section{ABSTRACT}

The objective of this work was to describe the perception of school coexistence from the viewpoint of the families of the students. As theoretical support some authors like Colombo (2011), Chaux (2013), Zaitegui (2006), Ramírez (2016), López de Mesa, Et al., (2013) and Valdes \& Urías (2010) were taken. Regarding the methodological aspects, the instruments were adapted from the ones proposed by Ortega and Del Rey (2002). The sample was non-probabilistic, the criteria for inclusion was being father of family of the students registered from first to fifth grade in the municipal official institution in Sincelejo- Colombia, also to assit to a general call, where only 58 family representatives made act of presence who are constituted in the sample. Data analysis was carried out through the use of descriptive statistics, the results show a favorable perception in the relationship between teachers and students, and however, among students the situation is different, presenting a high percentage of fights, nicknames and insults. And the breach for norms, are situations that disfavor the school climate, however, most conflicts are associated with a small group of students with disruptive violent behaviors and mistreatment, which affects the classroom climate. Faced with these situations, families suggest the need to implement a strategy for the integral improvement of coexistence that strengthens citizen competencies to guarantee an increase in the quality of coexistence that strengthens citizen competencies to guarantee an increase in the quality of coexistence.

Key words Coexistence, School, Family, Conflict.

\section{Introducción}

Siendo las instituciones educativas escenarios de interacción social, se presentan relaciones dinámicas complejas entre sus integrantes directivos, docentes, estudiantes y administrativos, y simultáneas a estas interacciones surgen situaciones que alteran la convivencia escolar. En el contexto de la investigación, la mayoría de los estudiantes 
provienen de familias de condiciones económicas similares, sin embargo se presentan diferencias entre las condiciones socioculturales, lo cual se refleja en las conductas y hábitos de los estudiantes.

Las diferencias socioculturales se constituyen en el foco de conflicto entre los estudiantes, debido a los contrastes en el comportamiento y los hábitos. Asimismo, en la participación de las familias se hace evidente estas diferencias en los procesos de acompañamiento escolar como reuniones, visitas a la escuela, acompañamiento de tareas escolares y acatamiento de reglas y normas.

La familia como primera célula de la sociedad, está convocada a participar de manera amigable en la crianza de sus hijos, sin embargo, no siempre esta participación contribuye al proceso de formación de los educandos. Para la realización de esta investigación participaron los asistentes, convocadas a través de una citación, a una reunión general para la básica primaria de una institución educativa oficial en Sincelejo-Colombia y corresponden al $38 \%$ de los padres o madres de familias. Esto corrobora que los sistemas de participación son distintos. Los resultados dan cuenta que las familias perciben como buena la convivencia escolar en la institución, puesto que consideran a nivel general la existencia de buenas relaciones entre los estudiantes y los docentes.

En consecuencia, los problemas y conflictos que se presentan en el ámbito escolar se encuentran según Ramírez y Arcilla (2013), de diversas formas "tanto física, moral, verbal, psicológica, material ofensivo o de intimidación (p. 420), que de alguna manera se vuelve inevitable su presencia en un espacio donde convergen miles de personas tal como ocurre en esta población objeto de estudio. Se considera que las altas tasas de situaciones de conflictividad en las escuelas colombianas pueden ser reflejo del contexto social existente desde hace muchos años, para Fuentes (2017) caracterizado por desempleo, desplazamiento forzado, pobreza, miseria y violencia generalizada tanto por grupos al margen de la ley (paramilitarismo y guerrilla) como bandas criminales y delincuencia común (p.1). 
Según diagnóstico institucional, algunos estudiantes habitan en familias con presencia de consumo de sustancias psicoactivas, violencia intrafamiliar, tribus urbanas, detrimento de valores, lo que conlleva a situaciones tensas y conflictivas que algunas veces pueden llegar a violencia, reflejándose de algún modo en el interior de las aulas. Por otro lado, es común encontrar cerca de la zona escolar billares, bares y moteles, y la presencia de varios núcleos familiares compartiendo el mismo espacio físico lo que connota hacinamiento.

Indudablemente, los procesos de enseñanza aprendizaje involucran para los educandos interacciones humanas, entre pares y adultos especialmente docentes, si bien, en las instituciones educativas, estas relaciones se encuentran condicionadas por normas y manuales, el componente afectivo como inherente al ser humano no debe obviarse completamente. Sin embargo, se aprecia en el ámbito del aula que muchos profesores y directivos restan importancia al afecto, como aspecto importante en el desarrollo del niño. En esta línea, Castro, Et al., (2011), consideran que, "el proceso de aprendizaje implica un componente afectivo que tiene influencia en las expectativas, motivación y funcionamiento académico" (p.196).

A partir del diagnóstico contextual de la institución correspondiente al año 2016, y el contacto directo con la población objeto de estudio, se tiene registro del poco apoyo y acompañamiento de las actividades escolares en casa por parte de las familias. Asimismo, se tiene conocimiento que los adultos son poco lectores y dedican el tiempo libre a actividades como ver televisión, escuchar música, juegos de azar entre otros

El tópico de la convivencia escolar ocupa un lugar importante en el desarrollo de la gestión escolar y del mejoramiento de la calidad educativa institucional. Al respecto, Ramírez (2016), plantea la convivencia escolar como "las acciones que permiten que los actores escolares puedan vivir juntos a través del diálogo, el respeto mutuo, la reciprocidad y la puesta en práctica de valores democráticos y para la paz” (p. 112). $\mathrm{U} n$ aspecto n e gat i vo en la convivencia escolar, es la violencia escolar. Sin embargo, "la violencia es algo evitable que obstaculiza la autorrealización, y por autorrealización 
humana se entiende la satisfacción de las necesidades básicas, materiales y no materiales"(Zaitegui, 2006: 437).

Por otro lado, la violencia escolar conforma una gran diversidad de conductas que se pueden dar entre los diferentes subsistemas que conviven en el contexto educativo (Colombo, 2011:11), de hecho, los procesos psicosociales y las normas que caracterizan las interacciones que se desarrollan en la escuela son lo que realmente diferencian una escuela de otra, en su configuración y en los logros obtenidos.

En este orden de ideas, la familia, es el primer espacio de socialización donde se crean las concepciones y explicaciones acerca de la convivencia y el ejercicio de los derechos. Es éste el lugar, donde el niño tiene las primeras experiencias de conflicto y comienza a conocer y a poner en práctica diferentes alternativas para enfrentarlos, resolverlos o evadirlos. Sin embargo, al parecer las familias no brindan las herramientas necesarias para la vida en sociedad. Pues, frecuentemente, presentan situaciones preocupantes dado que los estudiantes se están involucrando en actividades delictivas que pueden ser el inicio de trayectorias de criminalidad desde muy temprano en la vida" (Chaux, 2013: 40).

Se ratifica entonces, la función básica que cumple el contexto familiar, pues allí, inicia la formación en valores, normas, y el desarrollo de competencias ciudadanas como escucha activa, manejo de emociones, manejo del conflicto y empatía. Sin embargo, en el marco de esta investigación se hizo evidente la poca claridad que tienen las familias en las prácticas de crianza, esto conduce al incremento de la crisis de valores que se vive en la escuela. Por otro lado, el trabajo docente, se constituye en un proceso continuo y esencial. Al respecto, Halls \& Hord (2006), menciona que los docentes y directivos docentes tienen una influencia directa en la construcción de relaciones y el aprendizaje de estudiantes, dada su incidencia en las motivaciones, habilidades y condiciones de la comunidad educativa (Citado en Ministerio de Educación nacional, MEN, 2013:13).

Hoy por hoy, se evidencia una educación que enfatiza en derechos, pero no en deberes y responsabilidades, lo que ha conducido a un desequilibrio en las interacciones 
sociales. El hombre para desempeñarse en cualquier ámbito, necesita ejercer unas competencias para convivir de manera armónica y pacifica con sus semejantes, y para actuar de manera productiva en la cotidianidad son necesarias las competencias ciudadanas, que comprenden no solo las capacidades cognitivas, emocionales y comunicativas sino también la integración de los conocimientos y las actitudes en su desempeño social (Chaux, Lleras y Velásquez, 2004).

En Colombia se reglamenta por parte del MEN (2004), los estándares básicos de competencias ciudadanas quienes establecen tres ámbitos de aplicación las: ámbito de convivencia y paz; ámbito de participación y responsabilidad democrática, y el ámbito de pluralidad, identidad y valoración de las diferencias para la formación ciudadana. Estas dimensiones a su vez contienen competencias agrupadas por grado de escolaridad. (p.12).

Mediante el proceso de lectura de los estándares se logra identificar como el ámbito de convivencia y paz está relacionado con la vida en sociedad, las relaciones interpersonales e intergrupales y acciones prosociales para la prevención de la violencia. Mientras que, el ámbito de participación y responsabilidad democrática tiene que ver con la construcción colectiva de acuerdos, el análisis crítico de normas y leyes, y la transformación de contextos sociales a través de la elección democrática, tales como el gobierno escolar; y el ámbito de pluralidad identidad y valoración de las diferencias hace referencia a asuntos propios de una sociedad pluriétnica y multicultural como lo es Colombia.

Para el desarrollo de la presente investigación se planteó como pregunta guía del estudio ¿cuál es la percepción sobre la convivencia escolar de acudientes o familias de una institución educativa oficial del municipio de Sincelejo? para identificar categorías emergentes que permitan emprender planes de acción y de mejora en busca de la calidad educativa. Para ello, fue necesario emplear un cuestionario validado por Ortega y Del Rey (2002), administrado a los padres a manera de entrevista para la recolecta de la información.

\section{Metodología}


El presente trabajo investigativo tiene enfoque mixto; se emplean ambos paradigmas (cuantitativo y cualitativo) con supremacía del paradigma cuantitativo. En ocasiones en necesario el uso de una metodología mixta porque se corresponde a una visión holística para el estudio de un fenómeno. Para el caso particular una visión cuantitativa y cualitativa tiene como intención simbolizar lo más parecida posible la realidad estudiada. Esta fase contempla el acercamiento a la realidad y la inserción en la comunidad conocida por Murcia (1992), como un proceso de acercamiento al grupo seleccionado (Citado en Fuentes y Pérez (2018: 8).

La población está constituida por 1004 familias representantes de estudiantes de todos los niveles: preescolar, educación básica primaria y secundaria y educación media, vinculadas en una Institución oficial del Municipio de Sincelejo. De esta población se determina una muestra intencionada de (58) familias atendiendo criterios como: querer participar en las entrevistas y ser padre o acudiente activo de educación básica primaria. Según Hernández, Fernández y Baptista (2014) "la muestra es un subconjunto perteneciente a la población, que es delimitada y a su vez también es representativa de la misma" (p.171). Esta selección, está acorde con los objetivos de investigación y corresponde a los grados cuarto y quinto de educación básica primaria, los cuales fueron seleccionados a partir de los datos obtenidos desde la observación directa.

Para la realización del presente estudio se usó el cuestionario sobre convivencia escolar propuesto por Ortega y Del Rey (2002), aplicando la versión para padres, este instrumento está validado en diferentes países europeos por Gázquez, Et al., (2007), en su primera parte corresponde a la dimensión de percepción de la convivencia con siete (7) preguntas cerradas tipo escala Likert y cuatro niveles de respuesta bien, normal, regular y mal, la segunda dimensión conflictividad escolar con (10) preguntas cerrada con escala Likert con 4 niveles de respuesta siempre, muchas veces, a veces y nunca. A su vez, se complementa información con 
entrevistas sobre situaciones de conflictividad en la escuela y las estrategias de mejoramiento propuestas por las familias.

La observación participante, permite registrar las acciones de los actores en su cotidianidad desde los aspectos de la realidad en estudio y centrando la atención para abordar las interacciones que acontecen en las dinámicas del aula. No obstante, dada la diversidad de la información generada en esta investigación se utiliza diferentes herramientas para la sistematización de la misma.

El análisis del cuestionario se llevó a cabo usando estadística descriptiva, mientras que para la información derivada de las observaciones se utilizó el análisis de contenido que permite codificar y ordenar y datos en categorías analíticas del cual damos cuenta brevemente en este capítulo.

\section{Resultados y discusión}

La información se analizó mediante estadística descriptiva y análisis de contenido. Para el análisis estadístico se tuvo en cuenta tres aspectos contemplados en el cuestionario de entrevista: percepción de la convivencia, percepción de la conflictividad en la institución y estrategias de afrontamiento desde la familia. Además, para el análisis de contenido se codificó la información y recodifico desde las categorías emergentes.

Percepción de la convivencia, en general la familia percibe como buena la relación que se establece entre directivos, profesores y sus hijos (ver gráfico 1.). 


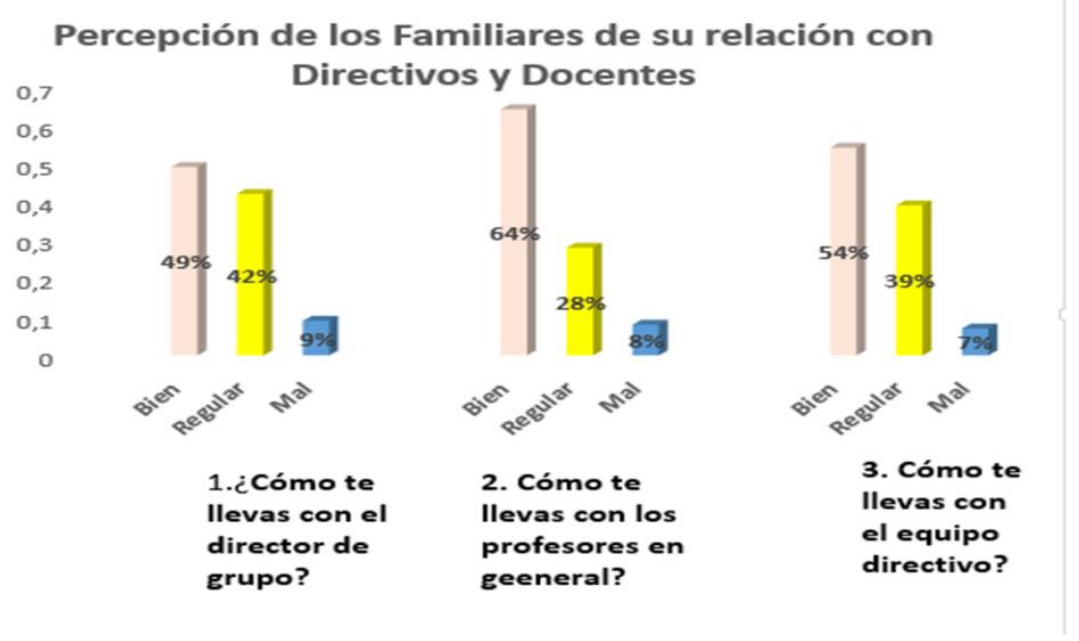

Gráfico 1 Percepción de los familiares de su relación con directivos y docentes Fuente: Elaboración propia.

El 54\% de los encuestados dice llevárselas bien con los directivos y docentes, pues ninguno manifestó malestar con alguno de los miembros del equipo directivo. Asimismo, es evidente que las familias se las llevan bien con los docentes representados en un $64 \%$ y dicen llevárselas bien con el director de grupo un $49 \%$. Esta información, da cuenta de un aspecto positivo de la convivencia puesto que, del comportamiento y actitudes de los padres depende en gran manera las relaciones de comunicación e interacción entre la escuela, los estudiantes y su familia (Palma y Álvarez, 2009).

Diversas investigaciones coinciden en afirmar que relaciones positivas entre los docentes y las familias inciden en el incremento del rendimiento académico. Hay más probabilidad que un estudiante con autoestima positiva, se esfuerce por superar dificultades, posea mayor interés por el aprendizaje y enfrente situaciones cotidianas con mayor asertividad. En esta línea, las interacciones sociales que se producen en las instituciones educativas para el desarrollo académico y la convivencia, se constituyen en un aspecto sustancial para la comunicación y el respeto entre sus miembros. Según López de Mesa, Et al., (2013), estas interacciones favorecen el desarrollo de habilidades 
sociales, permiten incrementar el aprendizaje y el rendimiento académico, así como la empatía, el aprendizaje cooperativo, la motivación escolar y la participación de los educandos en el proceso educativo (p. 385).

Sin embargo, es importante tener en cuenta que de 100 padres sólo el 52\% asisten a la invitación, estos corresponden precisamente a los que están más comprometidos con la crianza de sus hijos y tienen mayor participación, el ideal es que todos se vinculen puesto que, el contacto directo de los padres a la escuela posibilita unas relaciones sociales más positivas. En este sentido Pinheiro (2006), resalta el apoyo de los educadores, padres de familia y los pares para fortalecer las relaciones y aumentar la capacidad en la resolución de conflictos (Citado en López de Mesa, Et al., 2013: 388).

En consecuencia, los estudiantes que están relacionados con aquellas familias que no tienen un acercamiento positivo en la escuela se encuentren en alta vulnerabilidad, dado que es usual que estudiantes con pésimos resultados académicos busquen amistades fuera de la escuela, no lleguen a la institución aunque salgan de sus casas, lo cual incrementa el riesgo de establecer vínculos de amistad no favorables y adicción a los juegos de azar y sustancias psicoactivas, y como la relación con la familia no es cercana estos no perciben los cambios en la conducta. Además, al no poseer una buena relación con el profesorado y los directivos para conocer esta situación el problema puede llegar a trascender. 


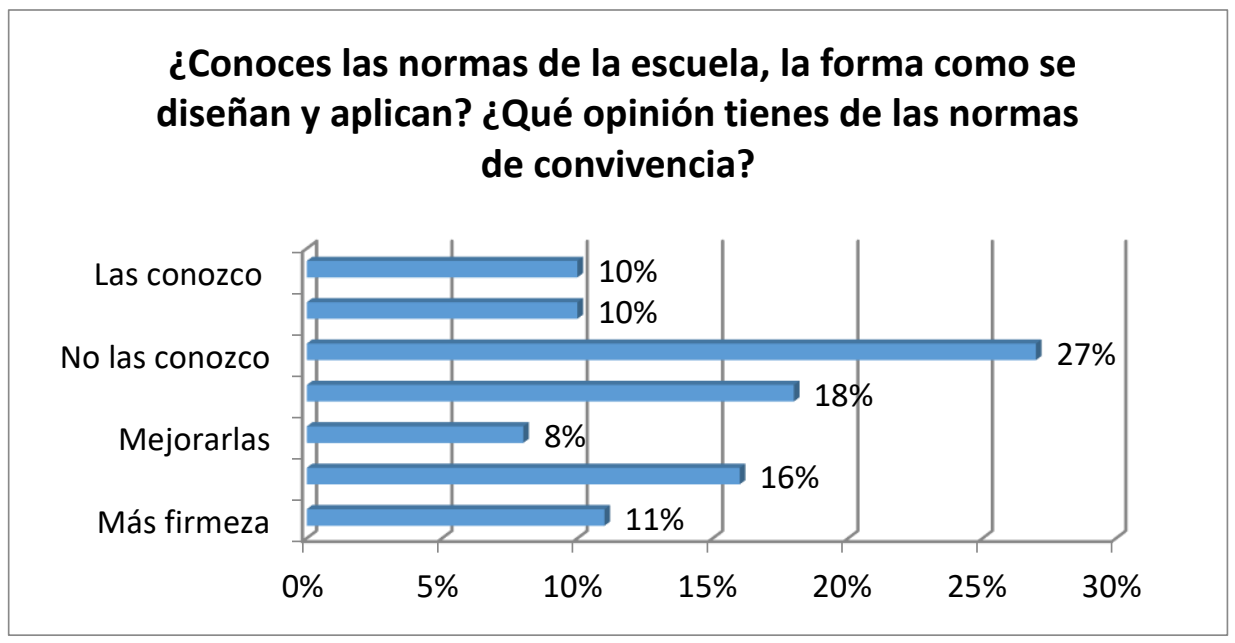

Gráfico 2. Percepción de las familias del conocimiento, diseño, aplicación y opinión de las normas de convivencia.

Fuente: Elaboración propia.

Por otro lado, el $27 \%$ de las familias encuestadas desconocen las normas establecidas en la escuela; un $18 \%$ de ellos afirman que estas normas no se respetan y un $16 \%$ opina que no se aplican. Sin embargo, existe un $20 \%$ que las conoce y las considera buenas; mientras que un $11 \%$ revela la necesidad de mayor firmeza en su aplicación y una minoría representada en el 8\% declara la necesidad de mejorarlas (ver gráfico 2.). Como se puede observar son innegables las discrepancias existentes en la percepción de la convivencia por parte de los representantes de las familias de la institución.

Por ello, se hace necesario fortalecer los vínculos entre las familias y la escuela, mediante la implementación de estrategias que conduzcan a un conocimiento más cercano de las dinámicas que se desarrollan al interior de ella, se disminuyan las brechas en esta relación para así, propender por una educación de calidad, pero, con la corresponsabilidad de las partes involucradas. De hecho, "el fortalecimiento de la 
relación familia escuela contribuye al desafío de una educación de buena calidad para niños y niñas” (Palma y Álvarez, 2009:5).

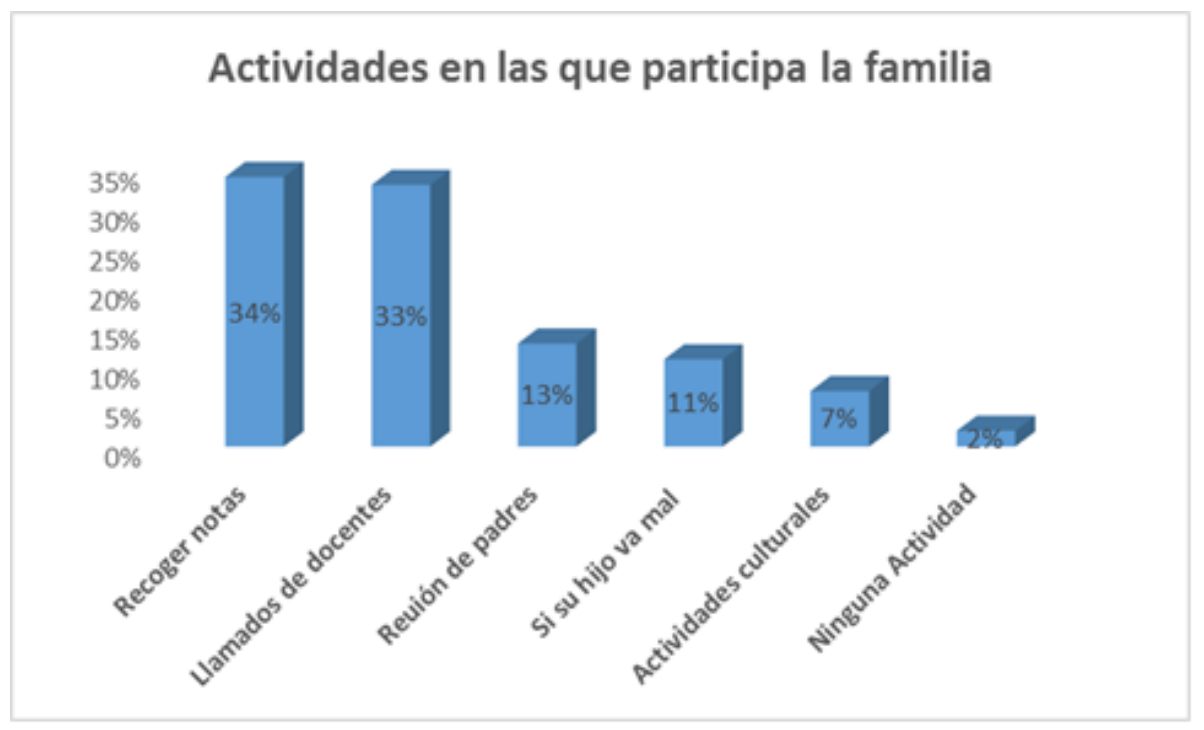

Grafico 3. Actividades en las que participa la familia

Fuente: Elaboración propia.

De otra parte, las familias dicen participar en las siguientes actividades recoger notas un 34\%, van si los llaman 33\%, reuniones de padres de familia 13\% si su hijo va mal $11 \%$ en las fiestas $7 \%$ y en nada $2 \%$, posiblemente el desconocimiento de las normas tiene que ver con su escasa participación, los padres encuestados no han sido participes de la construcción de normas y del manual de convivencia. Es probable, por tanto, que no hayan asistido a las reuniones en donde se hizo la divulgación de las mismas, en este sentido se empiezan a crear brechas dado que, si actores como los padres de familia desconocen las normas de convivencia, carecen de herramientas para orientar a sus hijos sobre su comportamiento en la escuela. La escasa participación por parte de acudientes y familias en los procesos escolares tienen que ver con que su interés 


\section{Luz S. Fuentes y Libia M. Pérez.}

Telos Vol. 21, No. 1 (2019). 61-85

está relacionado sólo con los logros académicos de los estudiantes Valdés y Urías (2010), (ver gráfico 3.).

\section{Percepción de la conflictividad}

Los resultados muestran que para las familias las situaciones conflictivas son menos aparentes, en este sentido, es primordial tener en cuenta que las percepciones de las familias se crean de manera indirecta, a partir de lo que escuchan por parte de los niños y los docentes (ver gráfico 4.).

Percepción de las familias sobre situaciones de conflictividad.

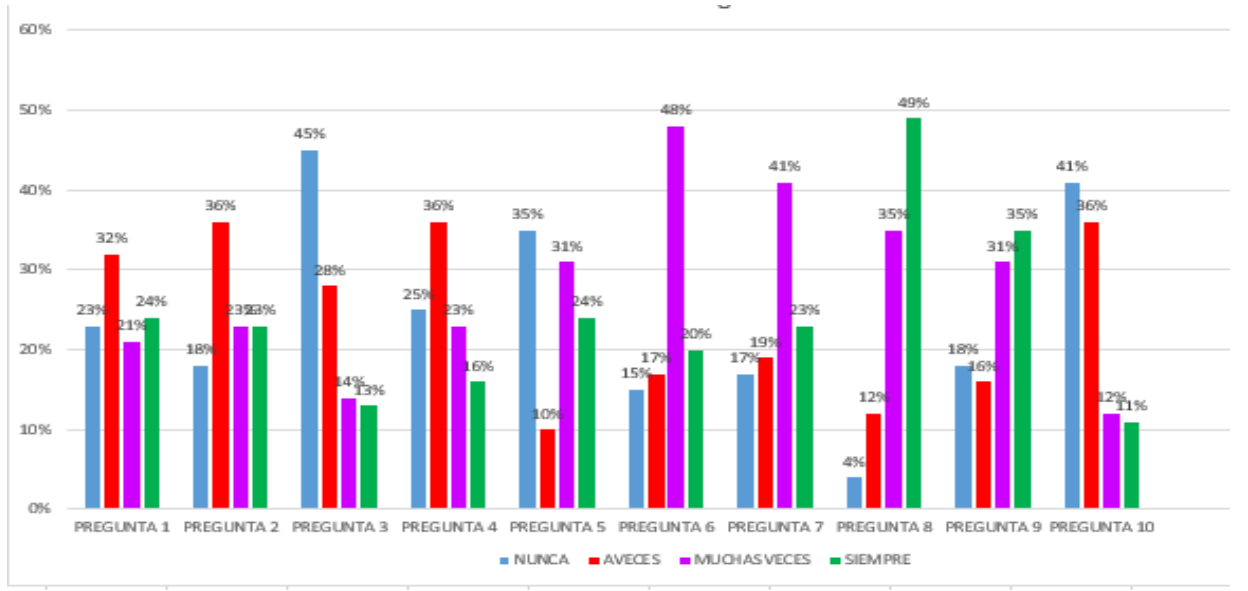

Gráfico 4: Percepción de las familias sobre las situaciones de conflictividad.

Fuente: Elaboración propia.

Respecto a la pregunta 10 ¿Hay enfrentamientos entre los estudiantes y el profesor?, las percepciones son variadas entre los padres un $41 \%$ dice que nunca se dan, un $36 \%$ manifiesta que a veces y el $23 \%$ restante se ubica en siempre y muchas veces, posiblemente estas diferencias están supeditadas a la gestión y liderazgo del orientador de grupo. 


\section{Convivencia escolar: una mirada desde las familias}

$\mathrm{Al}$ preguntarles a los padres si conocen sobre las malas contestaciones en clases por parte de los estudiantes (correspondiente a la pregunta 9), el 66\% dice que se presentan siempre y muchas veces, mientras que el resto expresa que a veces y nunca, lo cual da muestra de percepciones diversas. Es evidente, que las malas contestaciones se dan con frecuencia en las aulas de clases, no obstante que todos los niños no cuentan a la familia lo que sucede en el colegio y menos los que tienen confrontaciones, a pesar de que los docentes citen a los acudientes. Sin embargo, se puede decir que la mayoría de los estudiantes tienen conductas pro-sociales y respetuosas.

En concordancia con el respeto y cumplimiento de las normas (pregunta 8), los entrevistados muestran diversas percepciones, la más predominante se ubica en siempre $49 \%$ y $35 \%$ muchas veces y en a veces y nunca el resto de entrevistados un $16 \%$. Para estos padres o acudientes, el hecho de que las normas no se cumplan es una situación alarmante y ponen de manifiesto las diferencias en cuanto al respeto de las mismas en la época en que ellos fueron escolares. En este punto es importante considerar la diferencia en la interpretación del cumplimiento de normas. Cabe resaltar, que el enfoque educativo de competencias básicas en Colombia exige un cambio en el desempeño de los estudiantes Martín y Puig (2007) exponen que "aprender a vivir de forma democrática sólo se puede conseguir viviendo democráticamente” (p.91). Por tanto, la escuela está convocada a promover acciones que constituyan relaciones más amigables para la convivencia.

En cuanto a la existencia de insultos y maltrato verbal entre pares (pregunta 7), un $23 \%$ de la familia considera que esta situación se presenta mucho, el $41 \%$ dice que se presenta regularmente, el $19 \%$ indican que se presenta poco y un $17 \%$ afirma que no se presenta. Se hace evidente el tipo de relación que se establece en el aula, indicios de acoso entre iguales.

Por otro lado, en relación a la existencia de grupos que se llevan mal (pregunta 5), un $24 \%$ afirma que se presenta mucho, un $31 \%$ dice que se presenta de forma regular, un $10 \%$ afirma que se presenta poco mientras un $35 \%$ dice que estas situaciones no se 
presentan. La presencia de estos grupos está relacionada con la lucha de poderes o el deseo por sobresalir lo que conlleva a que sus interacciones sean conflictivas.

En este sentido, la cultura escolar adquiere especial relevancia en este contenido transversal, dado que transmite una serie de aprendizajes referidos al tipo de convivencia que propone y reproduce la escuela en sus prácticas cotidianas tales como formas de comportamiento, normas de conducta, valores aceptados o rechazados... (Ministerio de Educación de Chile, MEN. 2013:7).

Por otro lado, en relación, a los estudiantes que no están integrados y se siente solo (pregunta 4), los resultados indican que las familias creen en un $25 \%$ que esta situación no se presenta, un $26 \%$ piensa que se presenta poco, $23 \%$ dice que se presenta con regularidad y un 16\% afirma que se presenta mucho. Estos resultados varían con la percepción que al respecto tienen los estudiantes. Este hecho puede llegar a afectar el rendimiento escolar de los estudiantes. Diversas investigaciones sostienen que la participación de las familias junto con los docentes tras un objetivo común, repercute de manera esencial en el aprendizaje de los niños, en su desarrollo y finalmente en su éxito en la escuela (Gázquez, Pérez y Carrión, 2011).

En relación a la pregunta (2) "los estudiantes piensan que sus profesores no los entienden", un 18\% de las familias piensan que esta situación no se presenta, $36 \%$ dice que se presenta poco, $14 \%$ y un $46 \%$ dice que se presenta muchas veces. Estas percepciones son diferentes de las percepciones de los niños quienes con mayor frecuencia piensan que los profesores no los entienden De allí la importancia de la participación escolar en la escuela. La participación es dependiente de la cultura familiar, la ideología, y creencias. El compromiso con el seguimiento del aprendizaje de los hijos podría vincularse con la disponibilidad de capital cultural de la familia. (Carriego, 2010).

Por otro lado, respecto a la pregunta relacionada con la desmotivación y aburrimiento de los niños y niñas en la escuela (pregunta 1), el $23 \%$ dice que no se 
presenta nunca, $32 \%$ dice que poco, $21 \%$ dice regular y un $23 \%$ dice que se presenta mucho, y que estas conductas necesitan ser disminuidas al interior de la institución ya que pueden incidir en la motivación para estar en la escuela y por consiguientemente en el desempeño académico e integral de los educandos.

\section{Situaciones de Conflictividad descritas por la Familia}

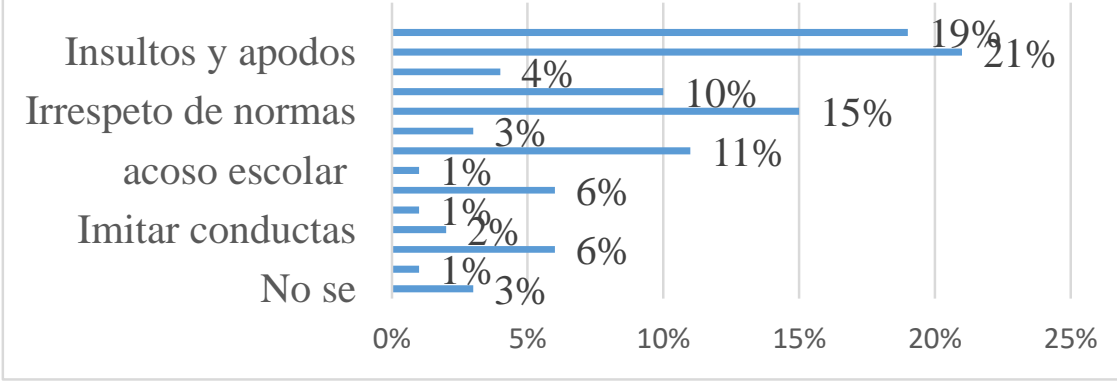

Gráfico 5. Situaciones de Conflictividad descritas por la Familia

Fuente: Elaboración propia.

En cuanto a las consideraciones de afrontamiento de la familia respecto a la conflictividad señalan los insultos y apodos en un $21 \%$ como la situación más generadora de conflicto, seguido de las agresiones físicas $19 \%$ y el irrespeto a las normas con un 15\%. (Ver gráfico 5). Se hace evidente entonces, la comunicación entre las familias y sus hijos pues las situaciones más perturbadoras de la convivencia escolar normalmente son comentadas en casa.

Del mismo modo, el mal clima escolar con presencia de gritos y ruido (11\%) así como la presencia de grupitos que no se llevan bien (10\%) también son situaciones contempladas por la familia, y la indiferencia del docente para mirar la conducta de los estudiantes $6 \%$ éstos últimos, aspectos los critican los familiares de los estudiantes, quienes se dirigen a los directivos docentes para manifestar sus quejas durante las entregas de notas. Puesto que, Pérez (2017), manifiesta como la violencia perturba de manera muy particular el ambiente escolar, y este afecta no sólo el desempeño 
académico sino la calidad de la enseñanza y perjudica las relaciones interpersonales (p. 239).

Otras de las situaciones contempladas por los padres de familia es la desmotivación de los estudiantes $4 \%$ y se sienten solos, 3\%; igualmente fueron considerados el acoso escolar, los hurtos y el enfrentamiento entre estudiantes con un $1 \%$. Por otro lado, $3 \%$ de los padres manifestó desconocer las situaciones conflictivas presentes. Estos resultados en la percepción de las familias indican que la participación de las familias en la escuela es muy escasa. Pero, en la actualidad se requiere que dicho vínculo refuerce la posibilidad de contribuir con la mejora de la escuela y que los distintos actores que conforman la comunidad educativa se comprometan con la calidad de sus procesos (Carriego, 2010: 65).

Estos datos se corresponden con la preocupación del Estado colombiano respecto a la poca participación del padre o acudiente en la relación con la escuela como educadora. Ante ello, aducen el no tener tiempo para vincularse en actividades escolares por los compromisos domésticos y labores externas que realizan. De tal modo, cuando son citados a la escuela, se acercan enojados pues, según ellos, los hacen perder el tiempo, si ésta no corresponde con la entrega de informe académico. Tal situación, requiere mejora desde la implementación de estrategias que vinculen a la familia de forma más directa a la escuela. Es decir, donde la familia se sienta importante en el proceso educativo y no sea ajeno a sus quereres y saberes la dinámica desarrollada al interior de la misma.

\section{Aspectos a mejorar en la convivencia}




\section{Aspectos mejorables en la Convivencia por las familias}

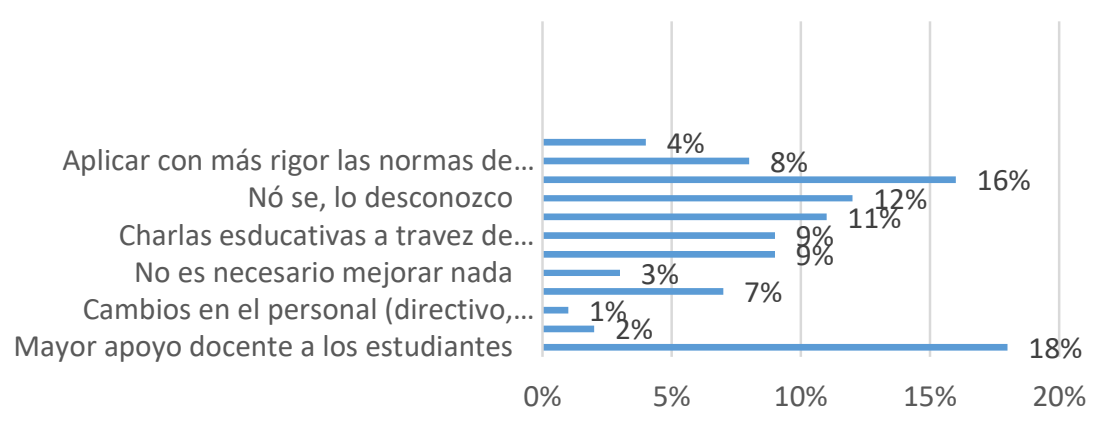

Gráfico 6: Aspectos mejorables en la Convivencia por las familias

Fuente: Elaboración propia.

Dentro de las actividades propuestas desde las familias para mejorar las situaciones de conflictividad resulta interesante que las familias piensan que las peleas o situaciones de conflictividad pueden ser mejoradas a través de una mayor participación de ellos (4\%). Esta apreciación coincide con lo que dice Moreno, Díaz, Cuevas, Nova y Bravo (2011) quienes opinan que la situación de conflicto disminuye cuando se cuenta como sustento sólido de la familia la cual le permite adaptarse a la escuela.

En este mismo orden, dentro de las actividades que se proponen por parte de la familia se encuentran: mayor apoyo docente a los estudiantes $18 \%$, mejorar el trato entre estudiantes $16 \%$, mejorar la información que entregan los docentes a las familias $11 \%$, incrementar charlas por parte de especialista a estudiantes $9 \%$, incrementar actividades recreativas $9 \%$, asimismo, existen familias que no conocen estrategias para mejoramiento un $12 \%$. Incentivar la comunicación entre los docentes, estudiantes y familia dentro del establecimiento educativo es garante de generar vínculos sanos y seguros para unas buenas relaciones interpersonales.

Por otro lado, es de conocimiento que la mayoría de los conflictos están asociados a un grupo reducido de estudiantes que tienen conductas disruptivas violentas 
y malos tratos, lo cual afecta el clima de aula. Ante esto, las familias consensúan una estrategia de mejoramiento integral de la convivencia que fortalecen las competencias ciudadanas, garantizando la buena convivencia, y el compromiso con actividades en las que la familia desea participar para ayudar a la convivencia.

Gráfico 7: Aspectos mejorables en la Convivencia por las familias

\section{Actividades en las que la familia desea participar para mejorar la convivencia}

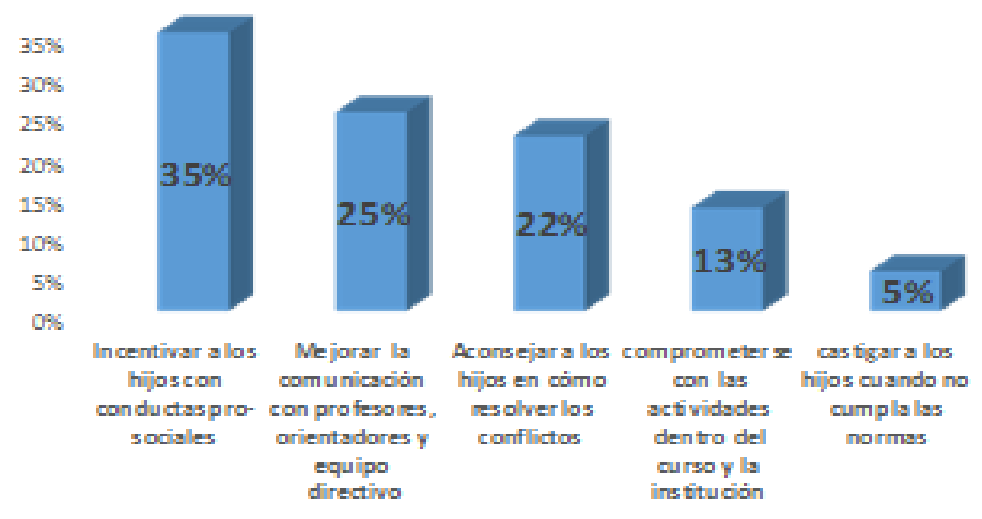

Fuente: Elaboración propia.

Dentro de ellas se encuentran principalmente incentivar a los hijos con conductas pro-sociales en un 35\%, mejorar la comunicación con profesores, orientadores y equipo directivo un $25 \%$, aconsejar a los hijos en cómo resolver los conflictos un $22 \%$, comprometerse con las actividades dentro del curso y la institución un $13 \%$ y castigar a los hijos cuando no cumpla las normas un 5\%. Estas actividades propuestas se constituyen en base para generar estrategias de mejoramiento para la convivencia escolar.

Producto de la entrevista efectuada a padres y/o acudientes en torno a como de desarrolla la labor de los docentes en la institución se logra evidenciar desde la visión 
del acudiente que los ambientes escolares son armónicos y la comunicación y armonía van de la mano.

Tabla 1. Labor de los docentes en la institución.

\begin{tabular}{|c|l|}
\hline Encuestado & \multicolumn{1}{|c|}{ Transcripción Textual } \\
\hline A1 & $\begin{array}{l}\text { Bueno realmente yo pienso que en muchas ocasiones los } \\
\text { docentes no realizan su trabajo en armonía ya que los } \\
\text { estudiantes constantemente se encuentran interrumpiendo } \\
\text { durante las clases y haciendo desorden. }\end{array}$ \\
\hline A2 & $\begin{array}{l}\text { Si yo creo que en general se trabaja muy bien. } \\
\text { Si por lo menos en lo que se alcanza a ver si, en mis hijos } \\
\text { sí, pero algunas veces si se observa que los estudiantes } \\
\text { están inquietos. }\end{array}$ \\
\hline A4 & $\begin{array}{l}\text { Para mí sí. Por el momento bien, yo creo que todo funciona } \\
\text { bien. }\end{array}$ \\
\hline A5 & $\begin{array}{l}\text { Bueno no tendría respuesta exacta porque yo } \\
\text { prácticamente vengo muy poco al colegio. }\end{array}$ \\
\hline A6 & $\begin{array}{l}\text { Yo creo que si porque yo veo que los docentes se llevan } \\
\text { bien a veces algunos problemas con los estudiantes pero } \\
\text { nada más. }\end{array}$ \\
\hline
\end{tabular}

Fuente: Elaboración propia

Dentro de la visión de los acudientes se observa que los ambientes escolares son armónicos. En general se trabaja bien, la comunicación y la armonía van de la mano, uno de los acudientes manifestó que los estudiantes interrumpen la jornada y alteran la normalidad académica. Esta situación de hecho fue manifestada por los docentes como muy molesta, pues las interrupciones constantes hacen que se pierda continuidad en el desarrollo de la clase. Cuando la clase no es continua sino interrumpida es necesario retomar explicaciones lo que disminuye la calidad en el proceso educativo (Peralta, 2004). 


\section{Tabla 2. Aspectos que debe cambiar o mejorar en la institución.}

\begin{tabular}{|c|c|}
\hline Encuestado & Transcripción Textual \\
\hline A1 & $\begin{array}{l}\text { Uno de los aspectos que deberían mejorarse en la institución es la } \\
\text { disciplina pues se observa que algunos de los chicos son muy } \\
\text { irrespetuosos entre ellos y hasta con los profesores. }\end{array}$ \\
\hline A2 & $\begin{array}{l}\text { Bueno yo pienso que el colegio como tal no, porque la mayoría } \\
\text { de los problemas vienen es de los niños y más que todo por lo que } \\
\text { ellos ven y viven en la casa. }\end{array}$ \\
\hline A3 & $\begin{array}{l}\text { Yo pienso que uno de los aspectos que se debe mejorar en la } \\
\text { institución es poner atención principalmente en el trato entre los } \\
\text { estudiantes porque yo he visto que realmente ellos se tratan muy } \\
\text { mal y eso afecta a todos los demás y también a las clases porque } \\
\text { los profesores les tocan andar interrumpiendo todo el tiempo. }\end{array}$ \\
\hline A4 & $\begin{array}{l}\text { Yo creo que todo está funcionando bien, de pronto se debe } \\
\text { cambiar un poco la agresividad de los niños y algunos papas } \\
\text { también. }\end{array}$ \\
\hline A5 & $\begin{array}{l}\text { Yo creo que debería cambiarse la disciplina porque antes el } \\
\text { colegio era casi perfecto entonces se debe cambiarse las normas } \\
\text { de disciplina y de convivencia por ejemplo algunas veces sucede } \\
\text { que alguno no trae el uniforme completo y lo dejan pasar los otros } \\
\text { inician a preguntar qué porque los dejan pasar, entonces si no } \\
\text { dejan pasar uno no deben dejar pasar a nadie. }\end{array}$ \\
\hline A6 & $\begin{array}{l}\text { Yo pienso que uno de los aspectos que debemos mejorar en la } \\
\text { institución es la convivencia porque algunas veces se observa } \\
\text { sobretodo en el descanso y en el patio que los estudiantes se tratan } \\
\underline{\text { muy mal entre ellos. }}\end{array}$ \\
\hline
\end{tabular}

Fuente: Elaboración propia.

Dentro de los aspectos visionados por los acudientes o familias que deben cambiarse tenemos: la disciplina, trato entre estudiantes y agresividad en la población estudiantil. Asimismo, se observa por parte de un acudiente una visión devaluada de 
la institución, con la existencia anterior de un mejor clima escolar, estas apreciaciones surgen porque muchos de los padres han tenido hijos mayores en la escuela o ellos mismos han sido estudiantes y por tanto tienen patrones de comparación. Desde Garreton (2013), la escuela por sus múltiples problemas de convivencia se convierte en un escenario que no garantiza un clima apropiado para que ella cumpla con sus objetivos.

\section{Conclusiones}

Los resultados revelan una percepción favorable en la relación entre profesores y estudiantes. Sin embargo, entre estudiantes la situación es diferente, pues se presenta un alto porcentaje de peleas, apodos e insultos. Situación que se controla dentro de la institución, pero, en ocasiones trasciende los muros de esta.

De igual forma, es notoria la exclusión y el irrespeto a las normas de comportamiento escolar, establecidas en el manual de convivencia y en los pactos de aula al inicio del año escolar; situaciones que desfavorecen el clima escolar. Conviene subrayar, que la mayoría de los conflictos están asociados a un grupo reducido de estudiantes con conductas disruptivas violentas y malos tratos, lo cual afecta el clima de aula. Las condiciones socioculturales al interior de la familia con predominancia de valores y normas claras son referentes de buena convivencia tanto al interior de la familia como en la escuela.

Las familias sugieren la necesidad de implementar una estrategia de mejoramiento integral de la convivencia que fortalezca las competencias ciudadanas en los estudiantes, para garantizar de esta forma la calidad en la convivencia escolar institucional.

La consecuencia de un conflicto no tratado a tiempo, desencadena variedad de violencia escolar, lo que implica trabajo conjunto entre las familias, el profesorado y el alumnado en la búsqueda de soluciones, pues, si no hay esa colaboración mutua, los logros no son los esperados. 


\section{Referencias Bibliográficas}

Castro, Marianella; Díaz, Mirta; Fonseca, Hilda; León, Ana; Ruiz, Lilian; y Umaña, Willy (2011). Las relaciones interpersonales en la transición de los estudiantes de la primaria a la secundaria. Revistra Electrónica Educare, Volumen 15, número 1. España. (pp. 193-210).

Carriego, cristina (2010). La participación de las familias: injerencia en la gestión y apoyo al aprendizaje. Revista Iberoamericana sobre Calidad, Eficacia y Cambio en Educación, REICE. Volumen 8, número 1. España. (pp. 50-67)

Colombo, Graciela (2011). Violencia Escolar y Convivencia Escolar: Descubriendo estrategias en la vida cotidiana escolar. Revista Argentina de Sociología, Volumen 8, número 9. Argentina. (pp. 81-104).

Chaux, Enrrique; Lleras, Juanita y Velásquez, María (2004). Competencias ciudadanas: de los estándares al aula. Revista de estudios sociales. Numero 19. Colombia. ( pp. 127-128).

Chaux, Enrrique (2013). Violencia Escolar en Bogotá: Avances y Retrocesos en cinco Años. Documento de Trabajo EGOB. Número 5. Extraído de https://ssrn.com/abstract=2926155 or http://dx.doi.org/10.2139/ssrn.292615 5. Consulta: 16-08-2018.

Fuentes,Luz Stella (2017). Formación en valores para el fomento de la convivencia escolar en instituciones educativas municipales. Doctorado en ciencias de la educación. Universidad Rafael Belloso Chacin. Venezuela.

Fuentes, Luz Stella y Pérez, Libia (2018). Perspectiva de los Estudiantes sobre la Convivencia Escolar. En Meriño, Víctor; Chirino, Yamaru; Camejo, Lineth y Martínez, Carmen (2018). Gestión del Conocimiento: Perspectiva Multidisciplinaria. Fondo Editorial Universitario Servando Garcés de la Universidad Politécnica Territorial de Falcón Alonso Gamero. Venezuela.

Garreton, Patricia (2013). Estado de convivencia escolar, conflictividad y su forma de abordarla en establecimientos educacionales de alta vulnerabilidad social de la provincia de concepción, chile. Doctorado en Ciencias de la Educación. Universidad de Córdoba. Chile.

Gazquez, José; Pérez-Fuentes, María y Carrión, José. (2011). Clima escolar y resolución de conflictos según el alumnado: Un estudio europeo. Revista de Psicodidática. Volumen 16, número 1, España. (pp, 39-58).

Gázquez, José., Cangas, Adolfo., Pérez-Fuentes, María., Padilla, David. \& Cano, Ángeles. (2007). Percepción de la violencia escolar por parte de los familiares: un estudio comparativo en cuatro países europeos. International Journal of Clinical and Health Psychology. Volumen 7, número 1. (pp. 93-105). 
Hernández-Sampieri, Roberto., Fernández-Collado, Carlos y Baptista-Lucio, Pilar. (2014). Metodología de la Investigación; Sexta edición. Editorial McGrawHill, México.

López de Mesa, Clara; Carvajal, Cesar; Soto, María y Urrea, Pedro (2013). Factores asociados a la convivencia escolar en adolescentes. Revista Educ. Educ. Volumen 16, número 3. (pp.383-410). Colombia. Extraído de http://educacionyeducadores.unisabana.edu.co/index.php/eye/article/view/271 6/3349 consultado el 11.05.2018 Consulta: 02-05-2018

Martín, Xus y Puig Rovira, Josep. (2007). Las siete competencias básicas para educar en valores. Editorial Graó. España.

Ministerio de Educación Nacional (MEN) (2013). Guía N 49: Guía Pedagógica Para La Convivencia Escolar. MinEducación. Colombia.

Ministerio de Educación Nacional (MEN) (2013). Gestión de la buena convivencia: Orientaciones para el Encargado de Convivencia Escolar y equipos de liderazgo educativo. MinEducación. Chile.

Ministerio de Educación Nacional (MEN) (2004). Serie Guía N6: Estándares básicos de competencias ciudadanas. Extraido de https://www.mineducacion.gov.co/1621/articles-75768_archivo_pdf.pdf

Consulta: 12-09-2017

Moreno, Cristian; Díaz, Alejandro; Cuevas, Carol; Nova, Carolina y Bravo, Ingrid (2011). Clima social escolar en el aula y vínculo profesor-alumno: Alcances, herramientas de evaluación, y programas de intervención. Revista Electrónica de Psicología Iztacala. Volumen 14, Número 3. Chile. (Pp. 70-84).

Ortega, Rosario y Del Rey, Rosario (2002). Los problemas de la Convivencia: desmotivación, conflictividad y violencia escolar. En Ortega, Rosario; Del Rey, Rosario y Gómez, Paula. Estrategias educativas para la prevención de la violencia. Mediación y diálogo. Edita, Cruz Roja Juventud. España.

Palma, Andrea y Álvarez Verushka (2009). Manual para profesores jefes. Acercando las familias a la escuela. UNICEF- Chile. Extraído de http://www.unicef.cl/web/wpcontent/uploads/doc_wp/Manual_Profes_Jefe.pdf consultado el 10.05.2018. Consulta: 20 -05- 2018.

Peralta, Francisco (2004). Estudio de los problemas de convivencia escolar en estudiantes de enseñanza secundaria, España. Tesis doctoral. Universidad de Málaga- España. Extraido de http://www.biblioteca.uma.es/bbldoc/tesisuma/16789222.pdf Consulta: 20 05- 2018. 
Pérez, Germarilin (2017). Manifestaciones y factores de la violencia en el escenario escolar. Revista Telos. Volumen 19, número 2. Venezuela, (Pp. 237-259).

Ramírez, Camilo y Arcilla, Wilian (2013). Violencia,conflicto y agresividad en el escenario escolar. Revista Educ. Volumen 16, número 3. Colombia. ( pp. 411249).

Ramírez, María (2016). Convivencia escolar y ambientes de aprendizaje en una escuela primaria de San Luis potosí. Revista International Journal. Volumen 2, número 2. Mexico. (pp. 111-126).

Valdés, Ángel y Urías, Maricela (2010). Creencias de padres y madres acerca de la participación en la educación de sus hijos. Revista Perfiles Educativos,

Volumen 33, número 134. México. (pp. 99-114).

Zaitegui, Nelida. (2006). La formación del Profesorado ante La violencia y la disciplina escolar. Ediciones Gráficas Arial, S. L. España. 